\title{
Challenges in the management of concurrent diabetic ketoacidosis and Dengue hemorrhagic fever in a child
}

\author{
V.Thadchanamoorthy ${ }^{1}$, Kavinda Dayasiri ${ }^{2}$, Dharshini Karuppiah ${ }^{3}$ \\ ${ }^{1}$ Faculty of Health Care Sciences, Eastern University, Sri Lanka \\ 2Base Hospital, Mahaoya, Sri Lanka \\ ${ }^{3}$ Teaching Hospital, Batticaloa, Sri Lanka.
}

\section{Abstract}

Diabetic ketoacidosis (DKA) is more commonly seen in children with type 1 diabetes and acute acidosis is usually precipitated by infections, trauma and surgery. Rarely, ketoacidosis can be precipitated by dengue often leading to management challenges in achieving haemodynamic stability. Occurrence of both conditions together warrant meticulous monitoring of fluid balance and control of blood glucose levels to manage both conditions optimally. Both type1 and type 2 diabetes upsurge the release of proinflammatory cytokines by various mechanisms and intensify the risk of plasma leakage. As prevalence of diabetes is lower in children compared with adults, diabetic ketoacidosis presenting with dengue is rare in the paediatric age group. We report a 14 year-old diagnosed child with type1 Diabetes mellitus who presented with dengue fever and subsequently developed dengue haemorrhagic fever and diabetic ketoacidosis concurrently. Both conditions were successfully managed with appropriate use of intravenous fluids and insulin. He had a complicated course with secondary bacterial infection needing intravenous antibiotics for 14 days. Ultimately he was discharged with his routine regimen of insulin and follow up was arranged at the endocrinological clinic. (SJDEM 2020/ Vol 10/ No 2)

Key words: Diabetic ketoacidosis, Insulin, Polyuria, Dengue

Correspondence email: v.vijayakumary@yahoo.com

Orchid ID: https://orcid.org/0000-0001-5361-7643

Copyright: This is an open-access article distributed under the terms of the Creative Commons Attribution License, which permits unrestricted use, distribution, and reproduction in any medium, provided the original author and source are credited (CC BY 4.0)

\section{Introduction}

Dengue is one of the most common tropical diseases in many countries including Sri Lanka. The disease has been a huge economic burden to the Sri Lankan health service at both primary and secondary care level over past two decades (1). There is a high incidence of comorbidity between dengue and diabetes especially in low-income tropical countries (2). Dengue fever similar to many other infections is known to precipitate diabetic ketoacidosis in patients with diabetes (3-5). Although the exact mechanism is not clear in both type 1 and type 2 diabetes, it is thought that cytokine mediated plasma leakage is complicated by diabetes $(6,7)$. Acute pancreatitis, which is an atypical complication of dengue, is also known to precipitate diabetic ketoacidosis (8). As children develop hyperglycemia during the acute stage, urine output becomes a less reliable indicator to guide fluid balance in management of patients with dengue haemorrhagic fever. Further, concurrent diabetic ketoacidosis has the potential to misguide the management of dengue shock and fluid management of diabetic ketoacidosis.
We report a child with poorly controlled type1diabetes in whom the management was complicated due to cooccurrence of diabetic ketoacidosis and dengue haemorrhagic fever.

\section{Case history}

A 14-year -old boy with type1 diabetes mellitus since the age of 8 years, who had overall poor control with recurrent hyperglycaemia and hypoglycaemia due to poor compliance to insulin injections, presented to the local hospital, with history of fever, severe headache, myalgia of 3 days, and abdominal pain and vomiting of 2 day duration. He was very thirsty and sweating excessively although he had adequate urine output more than $1 \mathrm{ml} / \mathrm{kg} /$ hour. He had no other features suggestive of respiratory or urinary tract infections and diarrheal illness. 
Physical examination revealed an ill-looking child and initial observations were: temperature- $39 \mathrm{C}$, heart rate 120 beats per minute, blood pressure - 110/60 mmHg, and respiratory rate $20 /$ minute. His pulse was of good volume. Investigations revealed leucopaenia (3.8x103 /cumm) and thrombocytopaenia (120x103 / cumm). Dengue NSI antigen was positive. Blood glucose level was $324 \mathrm{mg} / \mathrm{dL}$ but initial urine ketones were negative. He was managed as dengue fever with the targeted oral and intravenous fluids for initial 2 days following admission. As he developed high fever, severe abdominal pain, and urine output started to increase $(>2 \mathrm{ml} / \mathrm{kg} /$ hour$)$, he was transferred to intensive care unit (ICU) for further management.

On admission to ICU, he was very irritable with severe abdominal pain; had a pulse rate of 150 / minute whilst on intravenous fluids of $3 \mathrm{ml} / \mathrm{kg} /$ hour; and blood pressure was $90 / 60 \mathrm{mmHg}$. Investigations revealed progressive leucopaenia $(1.2 \times 103 / \mathrm{cumm})$ and thrombocytopaenia (70x103/cumm), Haemoglobin was $14.5 \mathrm{~g} / \mathrm{dL}$ with haematocrit being 44.5. C-reactive protein was $98 \mathrm{mg} / \mathrm{dL}$. Blood glucose went up to $524 \mathrm{mg} / \mathrm{dl}$ with positive ketones in the urine. Liver functions were also deranged (ALT-180 U/L, AST-224 U/L). Renal function was within normal limits except high blood urea $(80 \mathrm{mg} / \mathrm{dL})$ which was secondary to dehydration. Ultrasound revealed right side pleural effusion with mild ascites. Arterial blood gas showed metabolic acidosis with bicarbonate of $15 \mathrm{mmo} / \mathrm{l}$. He was diagnosed as having diabetic ketoacidosis during the critical phase of dengue hemorrhagic fever.

He was resuscitated with two $10 \mathrm{ml} / \mathrm{kg}$ boluses of $0.9 \%$ saline and one bolus of dextran 40 as pulse pressure dropped to $15 \mathrm{mmHg}$ indicating early shock. Vital signs such as pulse pressure, pulse volume and capillary refilling time were used to guide fluid status as urine output was not reliable. $\mathrm{He}$ was subsequently commenced $0.9 \%$ saline with soluble insulin $0.1 \mathrm{u} / \mathrm{kg} /$ hour and blood glucose was monitored one hourly until blood glucose levels stabilized between 200$300 \mathrm{mg} / \mathrm{dl}$. The dose of the insulin was reduced to half with the normal saline instead of adding dextrose due to risk of worsening of plasma leakage following hyperglycaemia. Fluid requirement was adjusted to between 5 to $7 \mathrm{ml} / \mathrm{kg} /$ hour to maintain minimum pulse pressure of $20-25 \mathrm{mmgHg}$ and maintaining capillary refilling time below 2 seconds. Fluids were in addition adjusted frequently to maintain vital signs as stable as possible. We were not guided by urine output which was consistently high during 36 hours of stay at the intensive care unit. Acidosis normalized with the correction of fluids and blood glucose levels.

As he had high fever and high C-reactive protein, he was started on intravenous antibiotics while being treated at the intensive care unit. He improved in 48hours following completion of the critical phase and he was able to start on oral fluids and food while on soluble insulin. His blood glucose normalized with negative urinary ketones 72 hours after he was admitted to ICU at which point he was changed to subcutaneous insulin with oral food and fluids and transferred to ward for further management.

As he had continued fever and high inflammatory markers, he was treated with a 14-day course of intravenous antibiotics. Blood and urine cultures were negative. Dengue specific IgM and $\operatorname{IgG}$ antibodies were positive. His hematological and biochemical parameters were within normal range at discharge from hospital. He was notified to national communicable disease surveillance system. He was reviewed in two weeks with fasting blood glucose and white cell count and both were within normal range. $\mathrm{He}$ was referred to the endocrinology clinic for long-term follow up. His HbA1c in 3 months while on insulin pen was $6.2 \%$. He was also referred to the dietician and psychologist for counseling regarding the importance of insulin and diet. His insulin dose was adjusted according to blood glucose level by the endocrinologist.

\section{Discussion}

We report an adolescent boy diagnosed with type1 diabetic mellitus, who presented with dengue fever and in whom, the course was complicated by occurrence diabetic ketoacidosis during the critical phase of dengue hemorrhagic fever. His clinical conditions improved with meticulous fluid management and intense monitoring during critical period of dengue haemorrhagic fever in an intensive care set up. Although there are reported cases of co-occurrence of dengue haemorrhagic fever and diabetic ketoacidosis in adults, presentation in paediatric age group is very rare. In this context, this report might improve awareness about challenges of managing both conditions in paediatric setting.

There are two contributory factors in the pathogenesis of co-occurrence of dengue haemorrhagic fever with type 1 diabetes. As type 1 diabetes is associated with autoimmunity, there will be continuous stimulation of the immune system leading to tissue inflammation, enhancement of leakage from capillaries and release of pro-inflammatory cytokines. The overall effect is high probability of leakage leading to dengue haemorrhagic fever and increased severity of dengue infection $(6,7)$. In addition, pathophysiology of dengue infection involves immune augmentation in the presence of previously acquired heterotypic antibodies against a serotype of the dengue virus at the time of new infection $(3,5)$. Both these factors promote the likelihood of dengue haemorrhagic fever by two and half times following dengue fever in patients with type 1 diabetes (4).

Although ideal total body water deficit among patients with diabetic ketoacidosis is $100 \mathrm{~mL} / \mathrm{kg}$ of body weight, the actual deficit becomes even higher with fluid loss in the phase of fluid leakage in dengue haemorrhagic fever due to leakage, and osmotic diuresis due to hyperglycemia. Therefore, continuous monitoring with 
adequate administration of intravenous fluids are crucial in the management of patients with concurrent dengue haemorrhagic fever and diabetic ketoacidosis. The initial fluid therapy would be isotonic solutions to maintain satisfactory tissue perfusion and urine output and it should be at least $0.5 \mathrm{~mL} / \mathrm{kg} /$ hour while insulin infusion is running. Intravenous fluids can be reduced slowly when the child has stable vital signs with return of appetite and polyuria (9). In our child, we had to give more fluid to maintain adequate pulse pressure. We were routed with pulse rate, volume, pulse pressure and capillary refilling time rather than urine output which had been more than expected due to osmotic diuresis. Cooccurrence of dengue haemorrhagic fever and diabetic ketoacidosis is very rare in children (2) and often challenging to the paediatricians. Delayed management can potentially lead to either under hydration resulting in dengue shock and severe metabolic acidosis or over hydration resulting in fluid overload or even cerebral oedema. The reported child presented early with dengue fever, and the probability of developing both dengue haemorrhagic fever and diabetic ketoacidosis was anticipated in advance. Early recognition resulted in optimal management of both conditions and prevented complications.

There were few case reports published on adults with similar clinical course ${ }^{(8,10,11)}$. One patient had acute pancreatitis as an atypical manifestation of dengue infection leading to transient diabetes. Once viraemia resolved, his diabetic state completely recovered (12).

\section{Conclusion}

Co-occurrence of diabetic ketoacidosis with dengue haemorrhagic fever in children is very rare. The rarity can potentially lead to delayed diagnosis of either condition accurately. Packed cell volume and haemodynamic parameters should be used to guide fluids as urine output is not reliable in indicating plasma volume status. Patients with concurrent diabetic ketoacidosis and dengue haemorrhagic fever are at high risk for shock due to fluid loss by both plasma leakage and osmotic diuresis. On the other hand, over-correction of fluids can lead to fluid overload or even cerebral oedema. Therefore, anticipation and early recognition are crucial for optimal management of concurrent diabetic ketoacidosis and dengue haemorrhagic fever.

\section{Acknowledgement}

The authors would like to thank Dr.A. Arulmuraly, Consultant Anaesthetist for helping to manage this patient.

\section{Conflict of interest}

The authors declare that there is no conflict of interests regarding the publication of this paper. 


\section{References}

1. Kumanan, Sujanitha V, Rajeshkannan N. The impact of population mobility on dengue fever: an experience from northern Sri Lanka, Sri Lankan Journal of Infectious Diseases 2019 ;(2):98102.DOI:http://doi.org/10.4038/sljid.v9i2.8252.https://doi.org/10.4038/sljid.v9i2.8252

2. Supradish PO, Rienmanee N, Fuengfoo A,et al. Dengue hemorrhagic fever grade III with diabetic ketoacidosis: a case report. J Med Assoc Thai. 2011; 94: 3:233-40.

3. Alexander Muacevic and John R Adler, Khemaporn Lertdetkajorn, Chutintorn Sriphrapradang et al. Management of Diabetic Ketoacidosis with Dengue Hemorrhagic Fever.Cureus. 2018; 10(9): e3336 2018; 10(9). doi: 10.7759/cureus.3336

4. Figueiredo MA, Rodrigues LC, Barreto ML, et al. Allergies and diabetes as risk factors for dengue hemorrhagic fever: results of a case control study. PLoS Negl.Trop Dis. 2010; 4:e699. https://doi.org/10.1371/journal.pntd.0000699

5. Kourí GP, Guzman MG, Bravo JR. Why dengue haemorrhagic fever in Cuba? An integral analysis. Trans $\mathrm{R}$ Soc Trop Med Hyg. 1987;81(5):821-3.https://doi.org/10.1016/0035-9203(87)90042-3.

6. Hsueh WA, Lyon CJ, Quiñones MJ. Insulin resistance and the endothelium. Am J Med. 2004; 117:109-17 https://doi.org/10.1016/j.amjmed.2004.02.042

7. Brown JM, Wilson TM, Metcalfe DD. The mast cell and allergic diseases: role in pathogenesis and implications for therapy. Clin Exp Allergy. 2008;38(1):4-18.https://doi.org/10.1111/j.1365-2222.1997.tb00665.x

8. Jusuf H, Sudjana P, Djumhana A, et al. DHF with complication of acute pancreatitis related hyperglycemia: a case report. Southeast Asian J Trop Med Public Health. 1998; 29:367-9.

9. World Health Organization. WHO Guidelines Approved by the Guidelines Review Committee. Geneva: WHO Press; 2009. Dengue: Guidelines for Diagnosis, Treatment, Prevention and Control: New Edition; pp. 1-144.

10. Fontal GR, Henao-Martinez AF. Dengue hemorrhagic fever complicated by pancreatitis. Braz J Infect Dis. 2011;15:4902.https://doi.org/10.1590/S1413-86702011000500015

11. Chen TC, Perng DS, Tsai JJ, Lu PL, Chen TP. Dengue hemorrhagic fever complicated with acute pancreatitis and seizure. J Formos Med Assoc. 2004;103:865-8.

12. Dalugama C, Gawarammana IB. Dengue hemorrhagic fever complicated with transient diabetic ketoacidosis: a case report. J Med Case Rep. 2017;11:302.https://doi.org/10.1186/s13256-017-1476-z 